\title{
DESARROLLO DE LA CIRUGÍA LAPAROSCOPICA: PASADO, PRESENTE Y FUTURO. DESDE HIPÓCRATES HASTA LA INTRODUCCIÓN DE LA ROBÓTICA EN LAPAROSCOPIA GINECOLÓGICA
}

\author{
Paolo Ricci A., Rodrigo Lema C., Vicente Solà D., Jack Pardo S., Enrique Guiloff F. \\ Unidad de Ginecología, Departamento de Obstetricia y Ginecología, Clínica Las Condes.
}

\section{RESUMEN}

Entendemos por endoscopia a aquellos procedimientos que permiten diagnosticar y tratar patología por medio de instrumentos con los que se observa el interior del cuerpo humano. Los primeros tratamientos por medio de endoscopia fueron realizados por Hipócrates. Luego a través de la historia son múltiples los precursores, pioneros y tantos otros que entregaron invenciones que permitieron llegar hasta el laparoscopio moderno. Convirtiéndose en una herramienta indispensable en la actual cirugía ginecológica mínimamente invasiva. Las nuevas tecnologías han permitido desarrollar la robótica, de la cual no han permanecido ajenos los procedimientos laparoscópicos. Así es como nace el robot Da Vinci, que más que una tecnología del futuro, ya constituye una realidad del presente en la cirugía laparoscópica ginecológica.

\section{PALABRAS CLAVES: Laparoscopia, cirugía mínimamente invasiva, cirugía robótica, Da Vinci}

\section{SUMMARY}

We understand by endoscope to those procedures that allow diagnosing and treated pathologies by means of instruments with which we observed in the interior of the human body. The first treatments by means of endoscope were made by Hipocrates. Soon through history the precursors, pioneers and so many others are multiple that gave inventions that allowed arriving until modern laparoscopy; becoming an indispensable tool in the present minimally invasive gynecological surgery. The new technologies have allowed developing the robotics technologies of which the laparoscopy procedures have not remained out. Thus the Da Vinci robot has born, who more than a technology of the future already constitutes a reality of the present in the gynecological laparoscopic surgery.

\section{KEY WORDS: Laparoscopy, minimally invasive surgery, robotic surgery, Da Vinci}

\section{INTRODUCCIÓN}

Desde los inicios de la medicina, el hombre se ha esmerado en crear instrumentos que le permitan diagnosticar y tratar patologías, sin la necesidad de realizar grandes incisiones. Así nace la endoscopia, cuyo principal característica, es la de utilizar técni- cas de mínima invasión. De esta manera se puede realizar una cirugía, con resultados cosméticos mejores, y con una recuperación más rápida, permitiendo un retorno lo antes posible a la vida actual (1). Sin embargo, se trata de una técnica quirúrgica que entrega una visión sólo en dos dimensiones, a través de una pantalla y sin sentido de profundidad. 
Además, muchas veces nos obliga a mantenernos en posiciones incómodas, por largo rato, con los brazos y cuello en extensión. Se trata de una técnica quirúrgica, que ha avanzado enormemente durante los últimos años, de la mano con el crecimiento de las nuevas tecnologías.

El desarrollo de la robótica, ampliamente utilizado en el proceso industrial, donde permite una mayor optimización de recursos y una mayor precisión, no ha quedado ajeno a la medicina. Es así como hoy en día se ha comenzado a utilizar exitosamente el robot Da Vinci, por medio del cual se realizan también cirugías ginecológicas. Esta nueva tecnología se presenta como de mayor precisión y comodidad para el cirujano. Sin embargo, evaluar los costos y la efectividad de la laparoscopia robótica es nuestra responsabilidad (1).

\section{Precursores de la endoscopia}

Entendemos por endoscopia al procedimiento por el cual observamos el interior del cuerpo humano mediante la introducción de instrumentos. Existe evidencia desde los inicios de la medicina, por el interés en crear instrumentos y procedimientos con este fin. El primero en realizar procedimientos tanto diagnósticos como terapéuticos a través de la endoscopia, fue el griego Hipócrates de Kos (460 AC370 AC). Se dice que utilizó cánulas para explorar en el interior de la boca y el ano; incluso habría llegado a tratar obstrucciones intestinales, a través de la insuflación de aire con esos instrumentos.

Por otro lado, existe evidencia que los romanos habrían utilizado diferentes tipos de espéculos, para lograr una visión a través de la vagina y el ano. Algunos de estos instrumentos fueron encontrados en las ruinas de Pompeya.

Muchos fueron los precursores de la endoscopia, que modificaron y crearon diferentes tipos de espéculos, entre ellos los ginecológicos. Instrumentos que sin duda fueron los primeros, en el intento por observar el cuerpo humano en su interior.

\section{Pioneros en laparoscopia}

El pionero de esta técnica fue Philip Bozzini (1773-1809), quien en 1805 presenta a la comunidad médica de Viena, su lichtleiter (Figura 1). Instrumento que permitía dirigir la luz al interior del cuerpo, obteniendo imágenes a través de la proyección y amplificación por medio de lentes y espejos. Sin embargo, su invento fue calificado como una "curiosidad" y fue amonestado por sus pares. Nunca llegó a utilizar su instrumento en pacientes, pero no hay duda alguna que los principios de su invento, sirvie- ron al desarrollo de la laparoscopia como la conocemos hoy.

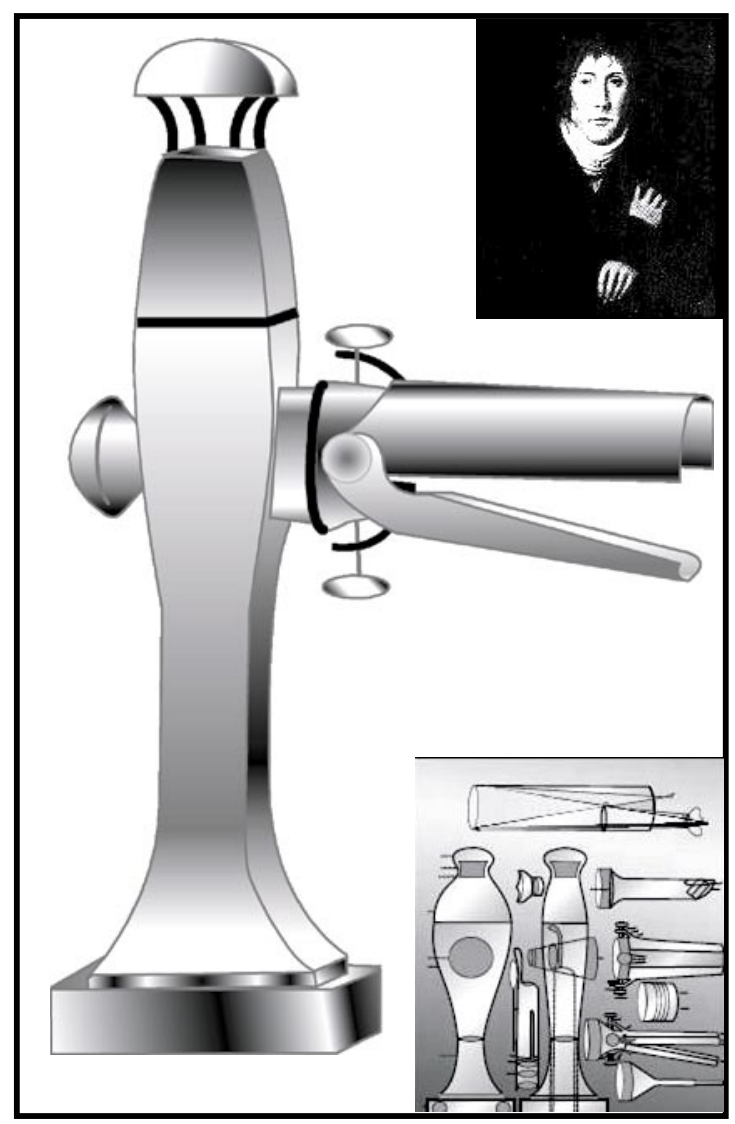

Figura 1. Lichtleiter de Bozzini (1805).

Fue sólo hasta 1853, cuando Antoine Jean Desormaux (1815-1870), en Francia, perfeccionó y utilizó por primera vez en humanos, el lichtleiter de Bozzini. La fuente de luz era una lámpara que hacía arder una mezcla de alcohol y turpentina (solvente tipo aguarrás). El mayor número de complicaciones se debía a las quemaduras de la fuente de luz. Este instrumento fue utilizado mayoritariamente en procedimientos urológicos. Desormaux es considerado el padre de la endoscopia. Sin embargo, no podemos dejar de mencionar que son muchos los colaboradores que en su mayoría, contribuyeron en forma independiente, con diferentes invenciones que permitieron llegar a la laparoscopia que utilizamos tan rutinariamente hoy. Entre ellos debemos destacar a George Kelling, que en 1901, en Berlin, inicia la utilización de un insuflador de aire, que filtraba por medio de algodones, con el fin de distender la cavidad abdominal para así detener sangrados por 
medio del pneumoperitoneo, lo que más tarde fue utilizado para lograr una mejor visión durante estos procedimientos.

\section{Beneficios de la laparoscopia en cirugía gineco- lógica}

Los médicos exigen cada día más a las empresas que proveen tecnología, para mejorar la calidad de vida de las mujeres a toda edad. Esto no es posible sin la introducción de tecnologías que permitan realizar cirugías sin el concepto de mínima invasión. Este concepto se debe apoyar y promover entre la comunidad médica (2). A diario las pacientes pueden aprovechar los beneficios de estas técnicas, en todos lo campos de la ginecología (3). La laparoscopia representa actualmente en forma integral la mínima invasión en cirugía ginecológica. La evidencia muestra que al comparar la cirugía por laparoscopia versus la laparotomía, la primera tiene una menor incidencia de complicaciones y una recuperación más rápida (4-6).

Durante el 2002, se publicó un meta-análisis, para comparar la seguridad de la laparoscopia versus la laparotomía en cirugía ginecológica (7). Se revisaron los estudios prospectivos, controlados, de Medline y la Base Cochrane, entre enero de1966 y junio del 2000. Se analizaron 27 trabajos, de cirugía por patología ginecológica benigna, completando 3611 mujeres (1809 laparoscopias y 1802 laparotomías). La laparoscopia se comprobó que tenía menos posibilidad de complicaciones generales (RR 0,59; 95\% IC 0,50-0,70).

La histerectomía por laparotomía tiene hospitalizaciones de 3 a 6 días y hasta 6 semanas de convalecencia. En cambio, la histerectomía laparoscópica tiene estadías hospitalarias de 1 o 2 días, con regreso total a las actividades entre 1 a 2 semanas (5).

La histerectomía laparoscópica permite y facilita el diagnóstico y tratamiento de patologías como endometriosis y adherencias. Facilita la remoción ovárica, la sección del ligamento ancho, la identificación de los uréteres, y permite realizar una hemostasia prolija (8). Por otro lado, la histerectomía abdominal permite al cirujano palpar los órganos pélvicos directamente. Sin embargo, puede aumentar la formación de adherencias y generalmente produce mayor dolor durante el postoperatorio. Además deja cicatriz en la pared abdominal. No necesariamente se obtiene una mejor visión de la cavidad abdominopélvica en algunas situaciones y patologías (8).

\section{Nuevas tecnologías en laparoscopia}

Sin lugar a duda, una de las tecnologías de gran importancia, incorporadas a la laparoscopia actual, es el láser. Este permite realizar con gran precisión disecciones de tejidos, controlando la profundidad de corte. En ginecología ha demostrado una gran utilidad en la cirugía laparoscópica de endometriosis, sobre todo al requerir la extirpación de zonas afectadas por esta patología en profundidad $(9,10)$.

Otra tecnología más recientemente incorporada, es el bisturí ultrasónico (11-13), que presenta ventajas sobre la electrocirugía (14). Permite realizar la disección de tejidos a través de energía ultrasónica, con una menor posibilidad de daño de estructuras vecinas, al no producir calor (15). El bisturí armónico tiene el potencial para convertirse en una de las energías preferidas para la realización de cirugías laparoscópicas (16). En ginecología se ha utilizado en todas las técnicas quirúrgicas, demostrando su efectividad, especialmente en la histerectomía (17).

Sin lugar a dudas, una nueva tecnología incorporada, que promete revolucionar la cirugía laparoscópica del futuro, es la robótica.

\section{Introducción de la robótica en la cirugía laparos- cópica}

La palabra robot se origina en el vocablo checo "robota", cuyo significado es "servidumbre, esclavitud, trabajo forzado". Fueron denominados de esta forma los trabajadores alquilados, que vivieron en el Imperio austro-húngaro hasta 1848. El término fue difundido en 1921, por Karel Čapek en su obra R.U.R. (Rossum's Universal Robots), en la cual el término fue utilizado para referirse a "humanos artificiales orgánicos". El significado actual de la palabra robot, fue definido por el Instituto de Robótica Americano (Robotic Institute of America) como aquella máquina que realiza funciones a semejanza del humano, pero con carácter mecánico y sin sensibilidad. Las primeras máquinas de las que se tiene registro con estas características, son las de Leonardo Da Vinci (1495) (18).

Con la explosión industrial actual, la robótica industrial ha tomado una gran importancia, al permitir que se efectúen trabajos de alta precisión en menor tiempo, a través de la automatización. Lo más utilizado es el brazo robótico, que tiene utilidad en todos los campos en los que se requiera realizar tareas repetidas, automatizadas, que requieran precisión o que pueden ser de peligro para los humanos. Más recientemente los robots han alcanzado también las tareas de la medicina. La primera cirugía con robots fue realizada en 1985, en el campo de la neurocirugía. Se trató del PUMA 560 que se utilizó para 
orientar una biopsia cerebral (19). Durante 1988 se utilizó el PROBOT (Integrated Surgical Supplies Ltd) en la resección prostática transuretral. En este modelo, se realizaba una presentación tridimensional de la próstata para luego calcular y efectuar los cortes $(20,21)$.

En 1992 la IBM (International Business Machine) desarrolló el ROBODOC, utilizado en cirugía ortopédica. Este robot fue utilizado por primera vez en el reemplazo de caderas $(22,23)$, y fue el primer robot aprobado por la FDA en cirugía. Este mismo año la NASA (National Aeronautics and Space Administration) solicitó a la compañía Computer Motion la creación de un brazo robótico para las tareas espaciales (Stanford Research Institute). En 1994 la FDA aprobó el uso del AESOP (Automated Endoscopic System for Optimal Positioning) $(24,25)$, desarrollado por la Computer Motion (Santa Barbara, California, EEUU). Se trataba de un brazo mecánico para sostener la cámara laparoscópica, lo que permitía reemplazar al ayudante en esta tarea. Los movimientos del brazo eran controlados manualmente o a distancia. Más tarde se agregó un control por voz $(26,27)$.

Otro sistema para el manejo de la cámara fue el ENDOASSIST, creado por Amstrong Health (High Wycombe, Reino Unido) $(28,29)$. La diferencia con el sistema AESOP, está en el control de los movimientos de la cámara a través de un sistema infrarrojo, accionado por los movimientos de la cabeza del cirujano, al observar el monitor.

El Departamento de Defensa de Estados Unidos, interesado en la posibilidad de realizar cirugías a distancia en posibles ambientes bélicos, impulsó el desarrollo de la telerrobótica. Este principio fue desarrollado bajo el proyecto denominado MASH (Mobile Advanced Surgical Hospital). Así nace el Da Vinci Surgical System, creado por la Integrated Surgical System (denominada en 1995, Intuitive Surgical, MountaZ in View, California, EEUU). Con este mismo propósito la compañía Computer Motion creó en 1998 el sistema ZEUS (sistema de control digital para operar a distancia los instrumentos laparoscópicos) $(30,31)$. Un sistema creado por la misma empresa, para el control del endoscopio mediante la voz del cirujano fue el HERMES $(32,33)$. A comienzos de 1999, ambas compañías, Intuitive Surgical y Computer Motion fueron acreditadas por la Comunidad Europea, para la utilización de sus sistemas robóticas Da Vinci y AESOP-Zeus. El primer sistema de visión en tres dimensiones para laparoscopia fue el ENDOWRIST (34) de Integrated Surgical System.

Durante el año 2000 la FDA (Food and Drugs
Administration) aprobó el uso de estos sistemas para EEUU. Aún cuando se trata de un sistema que permite realizar cirugías a distancia, la FDA aprobó utilizar los comandos de operación a distancia en la misma sala operatoria donde se encuentra la paciente.

Durante el 2001 la Computer Motion creó el sistema SOCRATES, que incluía equipamiento de telecomunicaciones. Con este sistema se realizó la primera cirugía transatlántica $(35,36)$. En junio de 2003, ambas compañías se fusionan, bajo el nombre único de Intuitive Surgical Inc., uniendo las tecnologías en el desarrollo del sistema integrado $\mathrm{Da}$ Vinci en el desarrollo la cirugía laparoscópica robótica (Figura 2 y 3). Más de 500 robots Da Vinci se encuentran operativos en diferentes hospitales en el mundo.

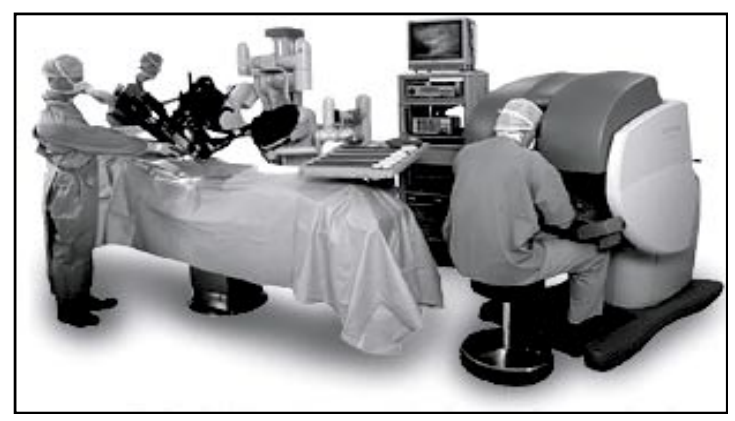

Figura 2. Sistema Integrado Da Vinci en un pabellón quirúrgico (2007, Intuitive Surgical, Inc)*.

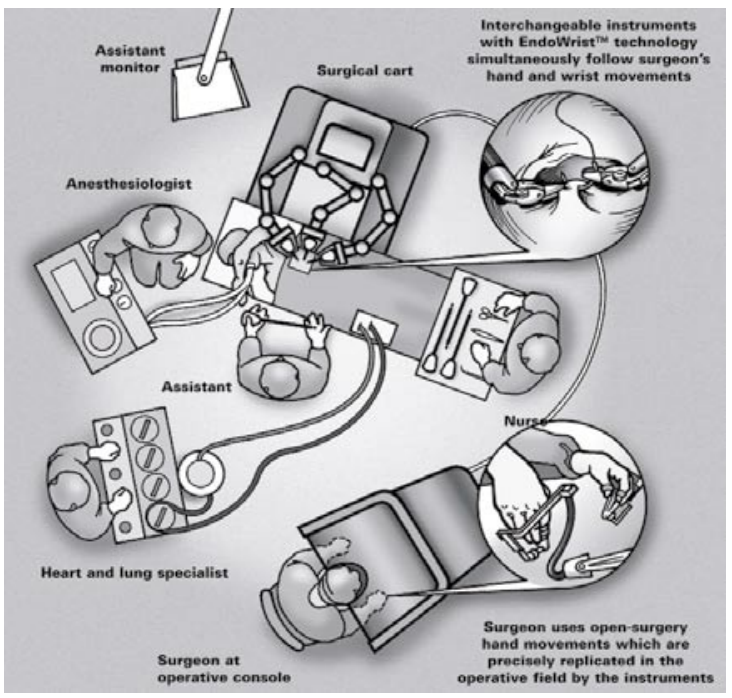

Figura 3. Esquema de la disposición del sistema Da Vinci y el personal en el pabellón de cirugía (2007, Intuitive Surgical, Inc) ${ }^{*}$. 


\section{Sistema integrado Da Vinci en laparoscopia}

El sistema Da Vinci combina la visión en tres dimensiones, con instrumentos con un rango de movilidad superior, lo que entrega un grado de precisión mayor. Además posee un sistema de control ergonométrico. Estas características permitirán una reducción del trauma causado por una cirugía, reducción de las pérdidas sanguíneas, menos dolor, recuperación más rápida, retorno a la vida laboral más rápido, mejores resultados cosméticos y una mayor precisión para realizar procedimientos de mayor complejidad. Todo esto a través de sus diferentes partes que lo forman:

A) Comando a distancia: se trata de un sistema que permite controlar a distancia los brazos del robot. Permite que el cirujano se apoye, sentado, aprovechando las características ergonométricas para realizar la cirugía. Está compuesto por una zona de visión, un mecanismo de control para ambas manos y pedales para accionar los diferentes instrumentos (Figura 4 y 5 ).

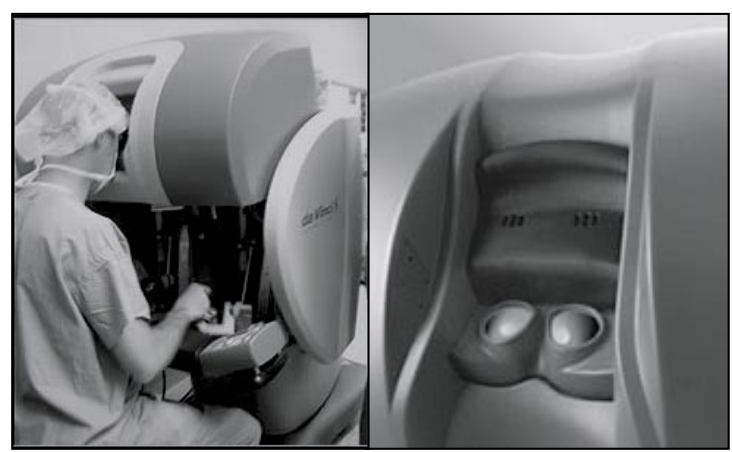

Figura 4. Sistema de comando a distancia del Da Vinci y detalle de la cabina con visión binocular (2007, Intuitive Surgical, Inc).

B) Soporte con brazos robóticos: consiste en una armazón que se coloca al lado de la camilla de la paciente, del cual salen 3 o 4 brazos robóticas, que sostienen y a través de los cuales se manejan a distancia los instrumentos específicos y la cámara, por medio de los cuales se realiza la cirugía (Figura 6). Los dos primeros brazos representan a la mano derecha y la izquierda respectivamente. El tercer brazo sostiene el endoscopio, que se controla desde la consola, por lo que no requiere otro cirujano para sostener la cámara. El cuarto brazo es opcional y puede ser utilizado en tareas adicionales.

C) Instrumentos específicos para la cirugía: se han diseñado con siete grados de movimiento, que imitan la destreza de la mano y muñeca humana. Esto

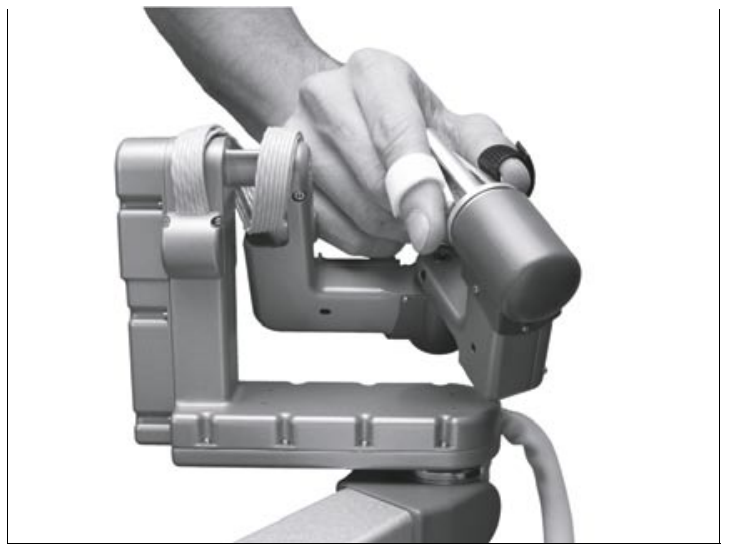

Figura 5. Detalle del sistema de manejo de instrumentos Zeus, ubicado en la consola de comando del robot Da Vinci (2007, Intuitive Surgical, Inc)*.

permite superar una de las desventajas de la laparoscopia tradicional, que posee un rango de movimiento limitado (37). Cada instrumento ha sido diseñado específicamente, permitiendo clampear, suturar o manipular los diferentes tejidos en los cuales se realiza la cirugía (Figura 7).

D) Sistema de visión: se trata de un sistema de alta resolución, que entrega una visión en tres dimensiones, magnificada. Este sistema incorpora la posibilidad de acercamiento instantáneo, controlado desde los comandos a distancia. Lo que permite al cirujano sentirse inmerso en el campo operatorio, entregando una mayor precisión para la manipulación de tejidos. Esto se complementa con un sistema de iluminación de gran intensidad. El telescopio es de $12 \mathrm{~mm}$ y posee en su interior dos cámaras de $5 \mathrm{~mm}$ cada una (Figura 8). Estas imágenes generan un campo operatorio virtual que pude observarse con una visión binocular en la consola de telecomando (Figura 4).

Una de las características de la tecnología aplicada en el diseño del comando a distancia es la de tener un diseño ergonómicamente superiores a cualquier instrumento construido con anterioridad para cirugía laparoscópica. Esto permite que el cirujano opere con mayor comodidad en todos los aspectos, incluyendo la visión.

El robot Da Vinci entrega al cirujano una tecnología jamás antes vista, controles ergonométricos avanzados, que permiten manipular a través de una visión de alta resolución en tres dimensiones, instrumentos diseñados con grados de movimiento, que imitan y superan al de la muñeca y mano humana. De esta manera, el cirujano dispone de herramientas que superan los beneficios de la cirugía laparos- 


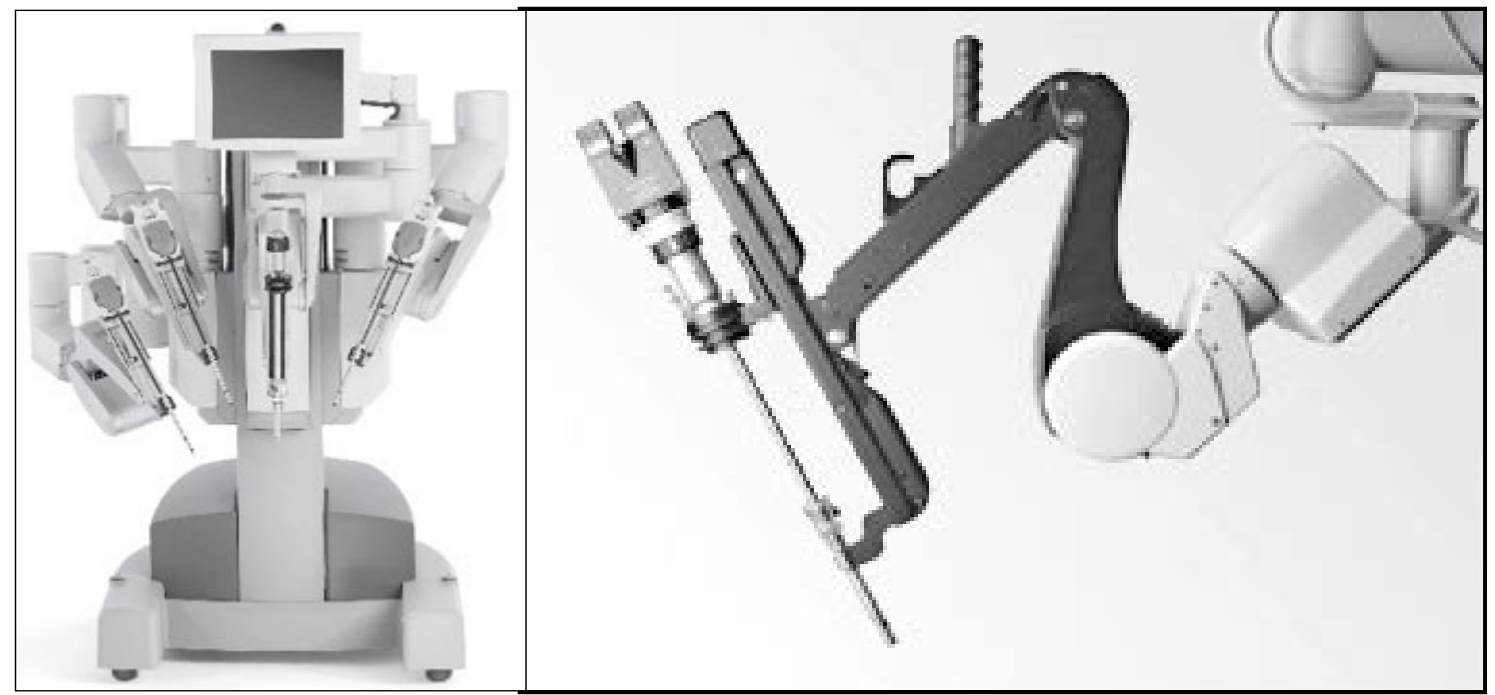

Figura 6. Sistema de brazos mecánicos. Detalle de un brazo (2007, Intuitive Surgical, Inc)*.

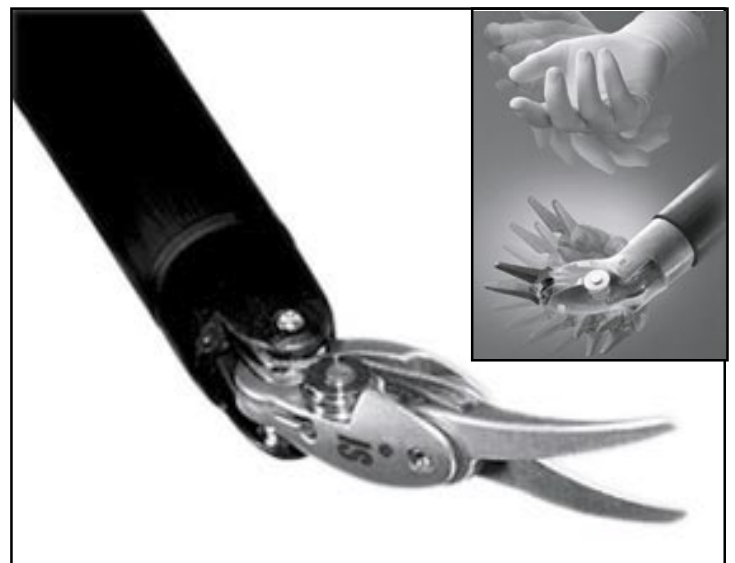

Figura 7. Detalle de una tijera y su amplia gama de movimientos que imitan y superan los de la muñeca y mano humana (2007, Intuitive Surgical, Inc)*.

cópica tradicional. Permitiendo realizar operaciones a través de trocares pequeños, con instrumentos de alta tecnología, que permiten una manipulación de mayor fineza y exactitud, con una menor posibilidad de daño de tejidos vecinos. Disminuyendo al máximo el posible daño neurovascular de estructuras vecinas. Por permitir una manipulación más exacta del campo operatorio, y reducir la posibilidad de daño por temblor propio del agotamiento de trabajar por horas con los brazos extendidos y gracias al sistema de corrección del temblor de manos, necesariamente reducirá el daño de tejidos vecinos, permitiendo mejores resultados quirúrgicos, con una recuperación y un retorno a la vida laboral más rápido. Por otro lado, contribuye a una mayor precisión la po-

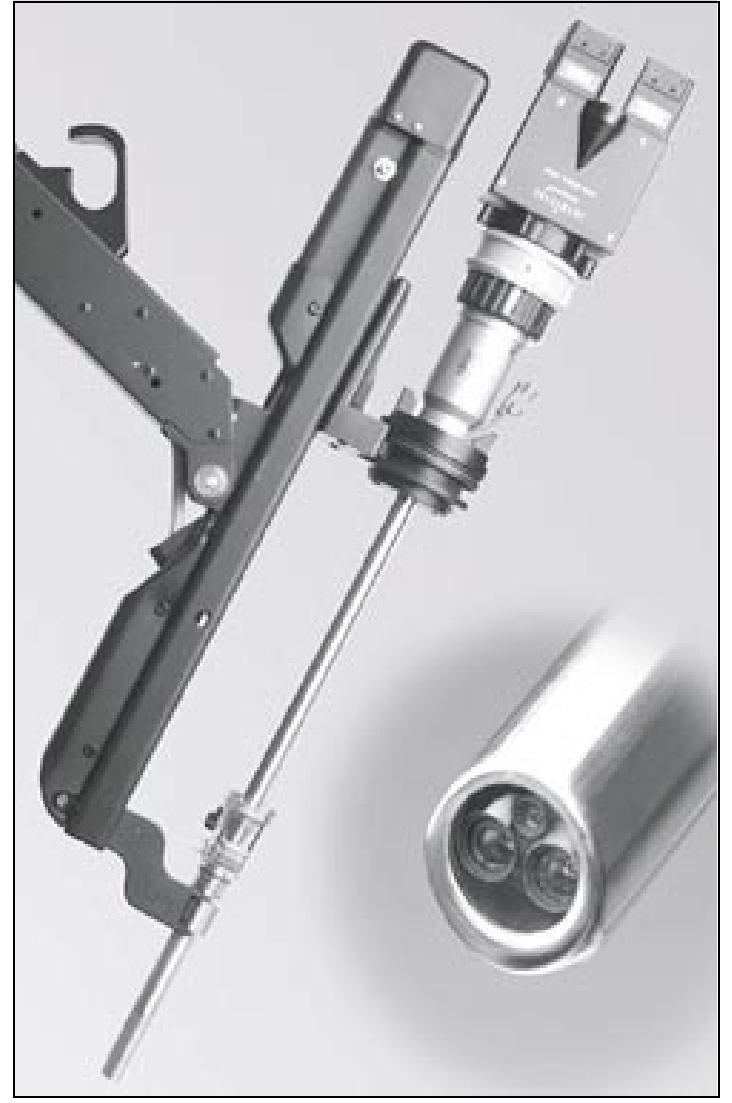

Figura 8. Cámara endoscópica con visión 3-D (2007, Intuitive Surgical, Inc)*. 
sibilidad de trabajar con instrumentos con grandes rangos y posibilidades de movimientos, a través de pequeñas incisiones de la piel, con instrumentos pequeños, con una imagen en tres dimensiones, de alta resolución y magnificada.

\section{Ventajas de la cirugía robótica (Tabla I)}

1. Telecirugía. La primera ventaja que surge de este sistema, es la posibilidad de realizar cirugías a distancia (telecirugía). Que fue el motivo que en primera instancia motivó el desarrollo de esta tecnología. El 7 de septiembre de 2001 se realizó la primera cirugía trans-oceánica. El cirujano estaba en Nueva York, EEUU, mientras que el paciente se encontraba en Estrasburgo, Francia. Se realizó una colecistectomía, con una transmisión que tenía un retraso de tan sólo 150 milisegundos (ida y vuelta de la señal), en una distancia de 7.500 kilómetros (38). Sin duda se trata de un caso extremo en lo que respecta a las distancias, pero demuestra la utilidad de la aplicación de esta tecnología ante la necesidad de realizar cirugías remotas. Actualmente, la tecnología de transmisión de datos, por vía satelital permite comandar a distancia $(39,40)$. Esto permitirá en el futuro aplicar la tecnología en situaciones y geografías extremas, como los polos, desiertos y lugares rurales. También en lugares afectados por catástrofes o guerras (41). Realizar cirugías en aviones o barcos acondicionados como hospital, que pueden trasladarse hasta estos lugares en estas condiciones, y duda, en el futuro próximo, con el desarrollo de la conquista del espacio, incluso realizar cirugías fuera de la atmósfera terrestre.

2. Mayor precisión. Dada por una visión estereoscópica que permite obtener una imagen en tres dimensiones, con aumentos de hasta 10 a 15 veces del campo operatorio (42). Además el procesador permite eliminar temblores o movimientos titubeantes innecesarios del cirujano (43). Otro factor importante es el disponer de instrumentos especiales, de mayor rango de movilidad que los de la laparoscopia tradicional.

3. Menor invasión. Esta tecnología cumple mejor aún el concepto de cirugía mínimamente invasiva que la laparoscopia tradicional. Permite realizar cirugías complejas a través de pequeñas incisiones, con instrumentos pequeños y de mayor precisión. Requiere un menor número y tamaño de aperturas en piel. Se realiza una pequeña incisión para la entrada de la cámara y dos para los instrumentos de trabajo. Sólo en caso necesario se puede utilizar el cuarto brazo, que está disponible para su utilización opcional, en los nuevos modelos del robot. Por otro lado, esta mayor precisión reduce al máximo el potencial daño de tejidos vecinos a las estructuras que necesitamos intervenir.

4. Mayor comodidad para el cirujano. La visión de alta resolución, en tres dimensiones, evita esforzar al máximo la visión, permitiendo realizar tareas quirúrgicas más complejas con mayor precisión y facilidad. Disminuye el estrés e incomodidad propia de realizar cirugías con imágenes de mala calidad, borrosas, y con posiciones viciadas, no naturales del cuerpo humano. El cirujano se encuentra sentado, en una posición más ergonométrica. En conclusión, el robot devuelve los grados de libertad perdidos en la laparoscopia convencional (44). Permite movimientos de los instrumentos que son similares e incluso superiores a los de la muñeca humana. Permitiendo un control a distancia, con instrumentos diseñados ergonométricamente, con la opción de manejo a través de las manos y/o de pedales (Figura 9). Si se requiere dejar un instrumento en posición fija para lograr por ejemplo, separar o sostener un órgano o tejido, no existirá el agotamiento $\mathrm{y}$ el temblor que secundariamente aparece como consecuencia de mantener por largo tiempo ciertas posiciones.

5. Permite obtener imágenes y la grabación de la cirugía, con alta resolución. Los paneles permiten incluso en control de imágenes digitales a través del tacto sobre la pantalla de monitores (touch screen). Esta ventaja, afecta directamente a la docencia e investigación, al permitir obtener material de alta resolución.

\section{Desventajas de la cirugía robótica (Tabla I)}

1. Costos. Toda tecnología nueva supone inversiones importantes en el proceso de diseño, planificación y creación. Por estos motivos los primeros modelos necesariamente serán los más caros. Sin embargo, la expansión del uso de este robot y el avance de la tecnología, permitirá abaratar los costos futuros.

2. Tiempo operatorio mayor. Este tiempo es dado en las diferentes publicaciones, por la implementación y preparación del robot, brazos e instrumentos. Necesariamente se debe realizar antes de comenzar la cirugía, el pneumoperitoneo y la introducción de los trocares, que van ajustados a los brazos mecánicos (45). Este tiempo de armado puede variar entre 14 y 27 minutos, mientras que el desarme entre 1 a 3 minutos (46). Indudablemente que este tiempo puede reducirse al adquirir la experiencia en el uso del robot. 


\section{Tabla I}

RESUMEN DE LAS PRINCIPALES VENTAJAS Y DESVENTAJAS DEL SISTEMA DA VINCI EN LA CIRUGÍA LAPAROSCÓPICA ROBÓTICA

\section{Ventajas \\ 1. Comodidad para el cirujano}

Consola de trabajo que permite una posición más ergonométrica, reduciendo el cansancio del cirujano a medida que transcurre una cirugía.

\section{Instrumentos}

Más pequeños, con mayores rangos de movimiento, l o que permite una mayor precisión y menos daño de tejidos vecinos.

\section{Mejor visión}

Permite I a visión en tres dimensiones, agregando el sentido de $p$ rofundidad que es una limitación en la laparoscopia clásica.

Imágenes de alta resolución, que se obtienen por un sistema de iluminación avanzado, con lentes que además permiten controlar la magnificación hasta 15 veces.

4. Reduce la posibilidad de daño a tejidos vecinos.

Posee un sistema de control del temblor de las manos del cirujano.

En conjunto las características que permiten una mejor visión y los instrumentos con mayores rangos de movimiento, permiten reducir el riesgo de daño, durante maniobras específicas que requieren mayor fineza de movimiento.

5. Posibilidad de realizar cirugía a distancia.

Aunque aún no se ha desarrollado masivamente, ya se han realizado cirugía con distancias transoceánicas.

Desventajas

1. Alto costo

Necesario debido al gran número de patentes y nuevas tecnologías desarrolladas, que sin embargo, una vez que se aumente su distribución, debería disminuir.

\section{Laparoscopia robótica en ginecología}

La cirugía laparoscópica ha revolucionado el concepto de cirugía mínimamente invasiva durante las últimas tres décadas. La cirugía asistida por robot ha sido una de las últimas innovaciones en el campo de la mínima invasión (46). Muchas técnicas quirúrgicas han sido realizadas mediante esta nueva tecnología, en especialidades como urología, cardiocirugía y cirugía general. La cirugía laparoscópica ha demostrado amplias ventajas sobre la cirugía por laparotomía (Tabla II). Sin embargo la laparoscópica asistida por robot, es superior a la tradicional (Tabla III). Durante el 2004 se estima que se realizó cirugía asistida por robot en más de 20.000 pacientes (47). La ginecología no ha sido la excepción, realizándose procedimientos en cirugía reproductiva, cirugía reconstructiva del suelo pélvico, ginecología general y oncología ginecológica. Algunas de estas cirugías son: miomectomía, tratamiento de endometriosis, ooferectomía, extirpación de quiste ovárico, colpopexia, Burch, histerectomía, 

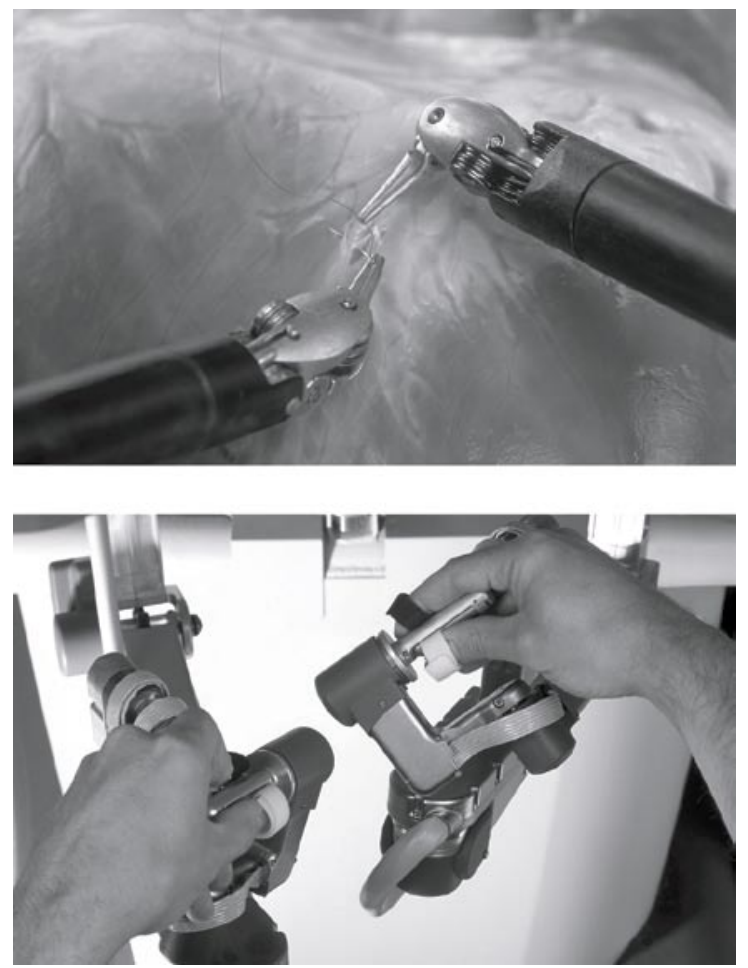

Figura 9. Detalle del manejo a distancia, mediante los controles Zeus, de un instrumento durante una sutura (2007, Intuitive Surgical, Inc)*.

salpingectomía, reanastomosis de las trompas de Falopio, transposición de ovarios y linfadenectomía (48-54). En el campo de la uroginecología, de gran desarrollo en los últimos años, se dice que la incorporación de mallas totales y la cirugía asistida por robot, son las mayores innovaciones (55).

Respecto a la histerectomía laparoscópica asistida por robot, primero fue realizada experimentalmente en animales (56). La primera publicación en humanos fue en 2002, por Diaz-Arrastia y cols (57). Actualmente la mayor parte de las publicaciones sobre experiencia en histerectomía laparoscópica asistida por robot, es sobre la clasificada por la AAGL (American Association of Gynecologic Laparoscopists) como tipo IVE y III. La evidencia actual demuestra la seguridad y efectividad de la robótica en cirugía ginecológica $(6,58-60)$.

Al ofrecer mayor precisión de trabajo, con instrumentos pequeños, con mayor rango de movilidad y visión en tres dimensiones, presenta ventajas sobre la laparoscopía tradicional. Permitiendo realizar histerectomías seguras, incluso en pacientes con fondos de saco anterior obliterados, que general-

mente son motivo de conversión a laparotomía en la laparoscopia tradicional (61). Sin lugar a duda, la tecnología laparoscópica robótica es superior a la convencional en la cirugía ginecológica por patología benigna y maligna (62).

\section{Tabla II}

\section{COMPARACIÓN DE VENTAJAS DE LA CIRUGÍA POR LAPAROTOMÍA VERSUS LA LAPAROSCOPIA TRADICIONAL}

Laparotomía

Ventajas

Visión real del campo

Visión de profundidad

Permite utilizar sentido de tacto

No requiere tecnología especial

\section{Desventajas}

Destrezas manuales limitadas a escala natural

Precisión limitada a instrumentos y visión

Sin posibilidad de aumento de visión de campo

Cansancio por posición del cirujano

Cicatriz mayor

Temblor de manos puede afectar la precisión

Recuperación más lenta post-operatoria

Menores resultados estéticos

Produce más dolor

\section{Reflexiones}

Hoy dependemos de un gran número de tecnologías, que sin duda alguna representan beneficios, para el paciente y los médicos. Sin embargo, y con toda la convicción de que los cambios deben ser a favor de las nuevas tecnologías que demuestren ser beneficiosas, no debemos olvidar que detrás de estas máquinas se encuentran personas: el médico y 


\section{Tabla III \\ COMPARACION DE VENTAJAS $Y$ DESVENTAJAS DE LA CIRUGÍA POR LAPAROSCOPIA TRADICIONAL VERSUS LA ASISTIDA POR ROBOT}

\begin{tabular}{|c|c|}
\hline Laparoscopia tradicional & Asistida por robot \\
\hline \multicolumn{2}{|l|}{ Ventajas } \\
\hline Mínimamente invasiva & Visión 3D \\
\hline $\begin{array}{l}\text { Tecnología bien } \\
\text { desarrollada }\end{array}$ & $\begin{array}{l}\text { Mayor posibilidad de } \\
\text { movimientos }\end{array}$ \\
\hline \multirow[t]{7}{*}{ Eficacia comprobada } & Diseño ergonométrico \\
\hline & Posible realizar telecirugía \\
\hline & Eliminación del temblø \\
\hline & $\begin{array}{l}\text { Mayor comodidad paa } \\
\text { el cirujano }\end{array}$ \\
\hline & Mayor precisión \\
\hline & $\begin{array}{l}\text { Permite realizar } \\
\text { micro-cirugía }\end{array}$ \\
\hline & $\begin{array}{l}\text { Permite gran magnificación } \\
\text { del campo }\end{array}$ \\
\hline \multicolumn{2}{|l|}{ Desventajas } \\
\hline Pérdida de sentido de tacto & Pérdida de sentido de tacto \\
\hline $\begin{array}{l}\text { Visión sin sentido de } \\
\text { profundidad }\end{array}$ & Tecnología de alto costo \\
\hline \multicolumn{2}{|l|}{ Movimientos limitados } \\
\hline \multicolumn{2}{|l|}{$\begin{array}{l}\text { Transmisión de temblor } \\
\text { del cirujano }\end{array}$} \\
\hline No ergonométrico & \\
\hline
\end{tabular}

el paciente. Si bien con estas nuevas tecnologías interactuamos con máquinas, no debemos olvidar lo importante que es la relación médico-paciente, base de todos los conocimientos actuales en medicina.

Con la creación de estas, cada vez más complejas tecnologías, y el avance específicamente de la computación, junto con adquirir estos costosos equipos, debemos garantizar un personal idóneamente entrenado, que sea capaz de actuar cuando se produzcan desperfectos. Toda máquina puede fallar y esta no será la excepción $(63,64)$. Entonces, a las complicaciones intraoperatorias, debemos agregar la falla del robot. Lo más probable que sea un solo equipo el que disponga cada hospital, para ser utilizado por las diferentes especialidades médicas, seleccionando a los pacientes de acuerdo a la complejidad quirúrgica requerida, por lo que si hay desperfectos que no sepamos solucionar o si no podemos cambiar las partes dañadas, no podremos continuar la cirugía, perdiendo todo el sentido de la utilización del robot.

El próximo paso será lo que podríamos llamar nanorobótica, es decir la reducción de los tamaños de los instrumentos, junto con el aumento de la complejidad de su funcionamiento, a favor de la obtención de mejores resultados quirúrgicos y la reducción de la posibilidad de complicaciones inherentes a la cirugía. De esta manera, sin duda se crearán pinzas o instrumentos de menor tamaño y con más funciones, porqué no, con una cámara y fuente de luz incorporada, que permitirán realizar cirugías con una aún "menor mínima invasión".

Esta tecnología podría incentivar al cirujano solitario, ya que al tener la posibilidad de conducir todo desde la consola de comando a distancia, en una posición más cómoda, podría sentir que no requiere de la intervención ni opinión de otros médicos. Sin embargo, debemos recordar que la discusión en conjunto de los casos, facilita el proceso que lleva a tomar decisiones correctas y acordes a cada paciente. Esta tecnología permite tener monitores, por medio de los que se puede permitir la observación, con la finalidad docente o simplemente para fomentar a otros que den su opinión.

Aún cuando la ciencia y tecnología avanzan a pasos cada vez más agigantados, lejanas parecen las tres leyes de la robótica, que fueron escritas por Isaac Asimov, y que él atribuye a John W. Campbell, aparecidas por primera vez la historia corta de ciencia ficción, titulado "Runaround". Escrita en octubre de 1941, publicada por primera vez en marzo de 1942 y contenida en la colección I Robot (1950), The Complete Robot (1982) y Robot Vision (1990).

1. Un robot no puede hacer daño a un ser humano o, por inacción, permitir que un ser humano sufra daño.

2. Un robot debe obedecer las órdenes dadas por los seres humanos, excepto si estas órdenes entrasen en conflicto con la Primera Ley.

3. Un robot debe proteger su propia existencia en la medida en que esta protección no entre en conflicto con la Primera o la Segunda Ley.

Los robots que actualmente se utilizan en cirugía laparoscópica asistida, corresponden a los llamados esclavos. Sin embargo, mientras se acerque cada vez más la posibilidad de la creación de inteligencia artificial, preferimos hoy recordarlas... 


\section{CONCLUSIONES}

Han sido muchos los precursores, pioneros e inventores modernos, quienes han permitido, a través de sus ideas e invenciones, el desarrollo de la laparoscopia hasta lo que conocemos hoy. No hay duda de los beneficios que la laparoscopia ha entregado en pos de la realización de mejores cirugías con mínima invasión. No hay duda que vendrán mejores tecnologías aún. Los años venideros prometen grandes avances, con instrumentos que permitirán entregar mayor precisión y calidad en el resultado de los tratamientos quirúrgicos. Sin embargo, la cirugía robótica, con todos sus beneficios, más que un futuro próximo, ya es un presente, que también ha alcanzado a nuestra especialidad.

\begin{abstract}
* Autorización de uso de las imágenes: The following photographic materials are provided here exclusively for promotion and/or media coverage of Intuitive Surgical and its products. This notification serves as an authorization for publications to make duplicate copies of the available high-resolution scans for editorial use only. http://www.intuitivesurgical.com/corporate/newsroom/mediakit/product_images.aspx
\end{abstract}

\section{BIBLIOGRAFÍA}

1. Romero Otero J, Paparel P, Atreya D, Touijer K, Guillonneau B. History, evolution and application of robotic surgery in urology. Arch Esp Urol 2007;60(4):335-41.

2. Adamyan LV. Minimally invasive surgery in gynecologic practice. Int J Gynaecol Obstet 2003;82(3):347-55.

3. Steiner RA, Fehr PM. Minimal invasive surgery in gynaecology. Ther Umsch 2005;62(2):127-38.

4. O'Hanlan KA, Lopez L, Dibble SL, Garnier AC, Huang GS, Leuchtenberger M. Total laparoscopic hysterectomy: body mass index and outcomes. Obstet Gynecol 2003;102(6):1384-92.

5. Shen CC, Wu MP, Kung FT, Huang FJ, Hsieh CH, Lan KC, Huang EY, Hsu TY, Chang SY. Major complications associated with laparoscopic-assisted vaginal hysterectomy: ten-year experience. J Am Assoc Gynecol Laparosc 2003;10(2):147-53.

6. Beste TM, Nelson KH, Daucher JA. Total laparoscopic hysterectomy utilizing a robotic surgical system. JSLS 2005;9(1):13-5.

7. Chapron C, Fauconnier A, Goffinet F, Bréart G, Dubuisson JB. Laparoscopic surgery is not inherently dangerous for patients presenting with benign gynaecologic pathology. Results of a meta-analysis. Hum Reprod 2002;17(5):1334-42.

8. Solà V, Ricci P, Pardo J, Guiloff E. Histerectomía: una mirada desde el suelo pélvico. Rev Chil Obstet Ginecol 2006;71(5):364-72.
9. McParland P, Halligan AW, Taylor DJ, Naftalin NJ. Laparoscopic laser treatment for endometriosis. Gynecol Obstet Invest 1997;44(1):38-40.

10. Sutton CJ, Ewen SP, Jacobs SA, Whitelaw NL. Laser laparoscopic surgery in the treatment of ovarian endometriomas. J Am Assoc Gynecol Laparosc 1997;4(3):319-23.

11. Gyr T, Ghezzi F, Arslanagic S, Leidi L, Pastorelli G, Franchi M. Minimal invasive laparoscopic hysterectomy with ultrasonic scalpel. Am J Surg 2001;181(6):516-9.

12. Lin J, Zhang X, Xu K. Application of ultrasonic scalpel in gynecologic operative laparoscopy. Chin Med J (Engl) 2001;114(12):1283-5.

13. Kauko M. New techniques using the ultrasonic scalpel in laparoscopic hysterectomy. Curr Opin Obstet Gynecol 1998;10(4):303-5.

14. McCarus SD. Physiologic mechanism of the ultrasonically activated scalpel. J Am Assoc Gynecol Laparosc 1996;3(4):601-8.

15. Kwok A, Nevell D, Ferrier A, Graf N, Lam A, Ford R. Comparison of tissue injury between laparoscopic coagulating shears and elctrosurgical scissors in the sheep model. J Am Assoc Gynecol Laparosc 2001;8(3):37884.

16. Solà V, Pardo J, Ricci P. Histerectomía mínimamente invasiva a través de laparoscopia con bisturí ultrasónico. Rev Chil Obstet Ginecol 2007;72(3):139-43.

17. HARMONICTM Ultrasonic Energy in Gynecologic Surgery: Hysterectomy with the HARMONIC ACETM and the McCarus Technique. McCarus SD. April 2006. Hallado en: http://www.obgmanagement.com

18. Dharia SP, Falcone T. Robotics in reproductive medicine. Fertil Steril 2005;84(1):1-11.

19. Padoan Junior AC, De A Barreto G, Araújo AF. Modeling and production of robot trajectories using the temporal parametrized self organizing maps. Int J Neural Syst 2003;13(2):119-27.

20. Davies B. A review of robotics in surgery. Proc Inst Mech Eng 2002;14(2):129-40.

21. Harris SJ, Arambula-Cosio F, Mei Q, Hibberd RD, Davies BL, Wickham JE, Nathan MS, Kundu B. The Probot--an active robot for prostate resection. Proc Inst Mech Eng [H] 1997;211(4):317-25.

22. Taylor RH, Joskowicz L, Williamson B, Guéziec A, Kalvin A, Kazanzides $P$, Van Vorhis R, Yao J, Kumar R, Bzostek A, Sahay A, Börner M, Lahmer A. Computer-integrated revision total hip replacement surgery: concept and preliminary results. Med Image Anal 1999;3(3):301-19.

23. Taylor KS. Robodoc: study tests robot's use in hip surgery. Hospitals 1993;67(9):46.

24. Nathan CO, Chakradeo V, Malhotra K, D'Agostino H, Patwardhan $\mathrm{R}$. The voice-controlled robotic assist scope holder AESOP for the endoscopic approach to the sella. Skull Base 2006;16(3):123-31.

25. Jacobs LK, Shayani V, Sackier JM. Determination of 
the learning curve of the AESOP robot. Surg Endosc 1997;11(1):54-5.

26. Allaf ME, Jackman SV, Schulam PG, Cadeddu JA, Lee BR, Moore RG, Kavoussi LR. Laparoscopic visual field. Voice vs foot pedal interfaces for control of the AESOP robot. Surg Endosc 1998;12(12):1415-8.

27. Johanet $\mathrm{H}$. Voice-controlled robot: a new surgical aide? Thoughts of a user. Ann Chir 1998;52(9):918-21.

28. Dagan J, Bat L. Endoassist, a mechanical device to support an endoscope. Gastrointest Endosc 1982;28(2):97-8.

29. Nebot PB, Jain Y, Haylett K, Stone R, McCloy R. Comparison of task performance of the camera-holder robots EndoAssist and Aesop. Surg Laparosc Endosc Percutan Tech 2003;13(5):334-8.

30. Zhou HX, Guo YH, Yu XF, Bao SY, Liu JL, Zhang Y, Ren $Y G$, Zheng $Q$. Clinical characteristics of remote Zeus robot-assisted laparoscopic cholecystectomy: a report of 40 cases. World J Gastroenterol 2006;12(16):26069.

31. Nio D, Bemelman WA, Balm R, Legemate DA. Laparoscopic vascular anastomoses: does robotic (ZeusAesop) assistance help to overcome the learning curve? Surg Endosc 2005;19(8):1071-6.

32. Panait L, Rafiq A, Mohammed A, Mora F, Merrell R. Robotic assistant for laparoscopy. J Laparoendosc Adv Surg Tech A 2006;16(2):88-93.

33. Luketich JD, Fernando HC, Buenaventura PO, Christie NA, Grondin SC, Schauer PR. Results of a randomized trial of HERMES-assisted versus non-HERMESassisted laparoscopic antireflux surgery. Surg Endosc 2002;16(9):1264-6.

34. Rassweiler J, Frede T, Seemann O, Stock C, Sentker L. Telesurgical laparoscopic radical prostatectomy. Initial experience. Eur Urol 2001;40(1):75-83.

35. Rassweiler J, Frede T. Geometry of laparoscopy, telesurgery, training and telementoring. Urologe $A$ 2002;41(2):131-43.

36. Rassweiler J, Frede T. Robotics, telesurgery and telementoring--their position in modern urological laparoscopy. Arch Esp Urol 2002;55(6):610-28.

37. Rassweiler J, Frede T. Geometry of laparoscopy, telesurgery, training and telementoring. Urologe $A$ 2002;41(2):131-43.

38. Marescaux J, Leroy J, Gagner M, Rubino F, Mutter D, Vix M, Butner SE, Smith MK. Transatlantic robot-assisted telesurgery. Nature 2001;413(6854):379-80.

39. Rayman R, Croome K, Galbraith N, McClure R, Morady R, Peterson S, Smith S, Subotic V, Van Wynsberghe A, Primak S. Long-distance robotic telesurgery: a feasibility study for care in remote environments. Int $\mathrm{J}$ Med Robot 2006;2(3):216-24.

40. Rayman R, Croome K, Galbraith N, McClure R, Morady R, Peterson S, Smith S, Subotic V, Van Wynsberghe A, Patel R, Primak S. Robotic telesurgery: a real-world comparison of ground- and satellite-based internet per- formance. Int J Med Robot 2007;3(2):111-6.

41. Latifi R, Muja S, Bekteshi F, Merrell RC. The role of telemedicine and information technology in the redevelopment of medical systems: The case of Kosova. Telemed J E Health 2006;12(3):332-40.

42. Damiano RJ, Tabaie HA, Mack MJ, Edgerton JR, Mullangi C, Graper WP, Prasad SM. Initial prospective multicenter clinical trial of robotically-assisted coronary artery bypass grafting. Ann Thorac Surg 2001;72(4):1263-8; discussion 1268-9.

43. Talamini M, Campbell K, Stanfield C. Robotic gastrointestinal surgery: early experience and system description. J Laparoendosc Adv Surg Tech A 2002;12(4):22532.

44. Villavicencio Mavrich $\mathrm{H}$. Da Vinci advanced robotic laparoscopic surgery: origin and current clinical application in urology, and comparison with open and laparoscopic surgery. Actas Urol Esp 2006;30(1):1-12.

45. Ruurda JP, Broeders IA, Simmermacher RP, Borel Rinkes IH, Van Vroonhoven TJ. Feasibility of robot-assisted laparoscopic surgery: an evaluation of 35 robotassisted laparoscopic cholecystectomies. Surg Laparosc Endosc Percutan Tech 2002;12(1):41-5.

46. Nezhat C, Saberi NS, Shahmohamady B, Nezhat F. Robotic-assisted laparoscopy in gynecological surgery. JSLS 2006;10(3):317-20.

47. Hashizume M. Robot-assisted surgery. Nippon Geka Gakkai Zasshi 2005;106(11):689-93.

48. Sert BM, Abeler VM. Robotic-assisted laparoscopic radical hysterectomy (Piver type III) with pelvic node dissection--case report. Eur J Gynaecol Oncol 2006;27(5):531-3.

49. Elliott DS, Krambeck AE, Chow GK. Long-term results of robotic assisted laparoscopic sacrocolpopexy for the treatment of high grade vaginal vault prolapse. J Urol 2006;176(2):655-9.

50. Advincula AP, Song A, Burke W, Reynolds RK. Preliminary experience with robot-assisted laparoscopic myomectomy. J Am Assoc Gynecol Laparosc 2004;11(4):511-8.

51. Degueldre M, Vandromme J, Huong PT, Cadiere GB. Robotically assisted laparoscopic microsurgical tubal anastomosis: a feasibility study. Fert Steril 2000;74(5):1020-3.

52. Reynolds RK, Burke WM, Advincula AP. Preliminary experience with robot-assisted laparoscopic staging of gynecologic malignancies. JSLS 2005;9(2):149-58.

53. Mao SP, Lai HC, Chang FW, Yu MH, Chang CC. Laparoscopy-assisted robotic myomectomy using the da vinci system. Taiwan J Obstet Gynecol 2007;46(2):1746.

54. Bocca S, Stadtmauer L, Oehninger S. Uncomplicated full term pregnancy after da Vinci-assisted laparoscopic myomectomy. Reprod Biomed Online 2007;14(2):2469

55. Novara G, Galfano A, Secco S, Ficarra V, Artiba- 
ni W. Prolapse surgery: an update. Curr Opin Urol 2007;17(4):237-41.

56. Margossian H, Falcone T. Robotically assisted laparoscopic hysterectomy and adnexal surgery. J Laparoendosc Adv Surg Tech A 2001;11(3):161-5.

57. Diaz-Arrastia C, Jurnalov C, Gomez G, Townsend C. Laparoscopic hysterectomy using a computer-enhanced surgical robot. Surg Endosc 2002;16(9):1271-3.

58. Advincula AP, Song A. The role of robotic surgery in gynecology. Curr Opin Obstet Gynecol 2007;19(4):3316.

59. Advincula AP. Surgical techniques: robot-assisted laparoscopic hysterectomy with the da Vinci surgical system. Int J Med Robot 2006;2(4):305-11.

60. Reynolds RK, Advincula AP. Robot-assisted laparos- copic hysterectomy: technique and initial experience. Am J Surg 2006;191(4):555-60.

61. Advincula AP, Reynolds RK. The use of robot-assisted laparoscopic hysterectomy in the patient with a scarred or obliterated anterior cul-de-sac. JSLS 2005;9(3):28791.

62. Magrina JF. Robotic surgery in gynecology. Eur J Gynaecol Oncol 2007;28(2):77-82.

63. Borden LS, Kozlowski PM, Porter CR, Corman JM. Mechanical failure rate of da Vinci robotic system. Can J Urol 2007;14(2):3499-501.

64. Field JB, Benoit MF, Dinh TA, Diaz-Arrastia C. Computer-enhanced robotic surgery in gynecologic oncology. Surg Endosc 2007;21(2):244-6. 